\title{
One-dimensional self-similar solution of the dynamics of axisymmetric slender liquid bridges
}

\author{
By D. RIVAS AND J. MESEGUER \\ Laboratorio de Aerodinámica, E.T.S.I. Aeronáuticos, Universidad Politécnica. Madrid. Spain
}

(Received 30 March 1983)

Liquid bridges appear in a large variety of industrial processes such as the so-called floating-zone technique, used in recent years in crystal growth and in purification of high-melting-point materials.

In this paper the dynamics of axisymmetric, slender. viscous liquid bridges having volume close to the cylindrical one, and subjected to a small gravitational field parallel to the axis of the liquid bridge, is considered within the context of one-dimensional theories. Although the dynamics of liquid bridges has been treated through a numerical analysis in the inviscid case. numerical methods become inappropriate to study configurations close to the static stability limit because the evolution time, and thence the computing time, increases excessively. To avoid this difficulty, the problem of the evolution of these liquid bridges has been attacked through a nonlinear analysis based on the singular perturbation method and. whenever possible. the results obtained are compared with the numerical ones.

\section{Introduction}

One-dimensional theories have not been used in Huid mechanie's to the same (extent that they have in solid mechanies. However. attempts have been made with reference to some spccific problems, mainly those in connection with the dynamics of both viscous and inviscid capillary jets (a scope of the state of the art in this fick an be found in Bogy 1979). Leaving aside the earlier work of llieber (1931). who generalized to viscous jets the results obtained by Rayleigh through a linear one-dimensional analysis. the one-dimensional inviscid slice model due to Lee $(19 i 4)$ must be pointed out. This model was derived under the assumption that. if the ratio of the perturbation wavelength to the undisturbed jet diameter is large enough. the axial relocity can be considered constant over each cross-section. Iore recently. an alternative one-dimensional model including viscosity effects was developed by (ireen $(1976)$ by using the basic theory of ane-dimensional Cosserat continuum. Both the sliee and the cosserat models have been used in liquid-bridge problems (.Meseguer 1983: Neseguer. Sanz \& Rivas 1983), although with the (osserat model only a linear analysis has been performed.

The problem under consideration in this paper is the determination of the processes involved in the evolution of a circular cross-section column of viscous liquid. held by surface-tension forces between two parallel. coaxial. equal-diameter solid disks. as shown in tigure 1. In earrying out the analysis the following assumptions are introduced : it is assumed that the properties of both the liquid (density and viscosity) 


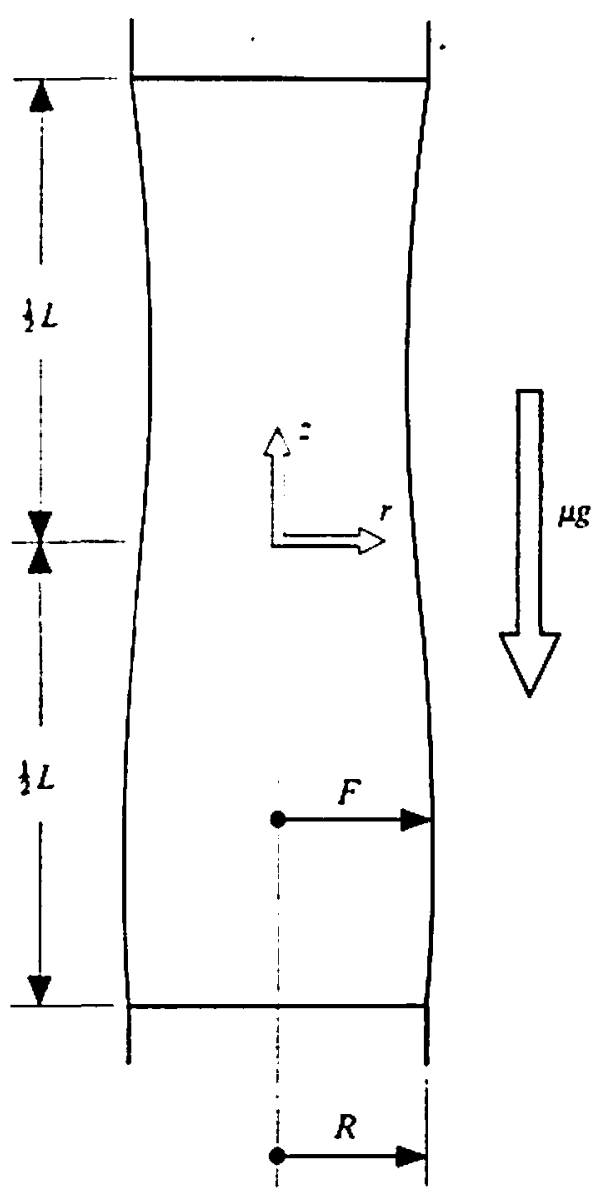

FinikE 1. (ieometry and coordinate system for the perturbed liquid bridge.

and the interface (surface tension) are uniform and constant, and that the effects of the gas surrounding the liquid bridge are negligible. Furthermore, it is assumed that inertia effects, due to a non-uniform displacement of the liquid column as a whole, are absent (the morement in the liquid is only due to the capillary-pressure gradient generated by the deformation of the interface and the hydrostatic-pressure gradient). Finally. since only axisymmetric configurations are considered, the problem is assumed to be independent of the azimuthal coordinate.

Under the above assumptions the liquid-bridge problem becomes suitable to be treated through one-dimensional theories, although a slight modification is needed aiming to introduce gravitational effects in the Cosserat model calculated by Green (1976).

\section{Problem formulation. One-dimensional Cosserat equations}

In the following all physical quantities are made dimensionless using the characteristic length $R$ (radius of the disks) and the characteristic time $\left(\rho R^{3} / \sigma\right)^{\underline{\ddagger}}$, where $\rho$ and $\sigma$ are the liquid density and surface tension respectively. Let $F(z, t)$ and $W(z, t)$ represent the liquid-bridge radius and the axial velocity at section $z$ and time $t$; the complete set of one-dimensional Cosserat equations is :

(a) continuity equation

(b) axial momentum equation

$$
\left(F^{2}\right)_{l}+\left(F^{2} W^{\prime}\right)_{2}=0
$$

$$
F^{2}\left(W_{\ell}+W^{*} W_{z}^{*}\right)=-\mathscr{P}_{2}+\left(F^{2}\right)_{z} P+2 C\left(F^{2} W_{z}\right)_{z},
$$

(c) radial momentum equation

$$
{ }_{8}^{1} F^{4}\left[\left(W_{t}+W_{z}\right)_{z}-\frac{3}{2} W_{z}^{2}\right]=-\mathscr{P}+F^{2} P+\frac{1}{8} C\left[\left(F^{4} W_{z z}\right)_{z}-8 F^{2} W_{z}\right] .
$$


Here $\mathscr{P}$ is an arbitrary function of $z$ and $t$, which will be removed from the formulation. $P$ accounts for both the capillary and hydrostatic pressure, and $C$ is a non-dimensional parameter giving the ratio of viscous to capillary forces: $C=\nu(\rho / \sigma R)^{\frac{1}{2}}, \nu$ being the kinematic viscosity of the liquid.

The formulation must be completed with boundary conditions, the axial velocity must be zero at the disks and the interface anchored to the disk edges:

$$
W( \pm \Lambda, t)=0, \quad F( \pm \Lambda, t)=1
$$

$\Lambda=L / 2 R$ being the slenderness of the liquid bridge. Finally, suitable initial conditions must be fixed:

$$
W(z, 0)=W_{0}(z), \quad F(z, 0)=F_{0}(z),
$$

Equations (2.2) and (2.3) can be reduced to one single equation by elimination of $\not 3$ between (2.2) and (2.3). If we choose as working variables $S=F^{2}$, which represents the cross-sectional area at $z, t$, and $Q=F^{2} W$, which is proportional to the axial momentum of a slice. the above formulation becomes

where

$$
\begin{gathered}
S_{t}+Q_{z}=0 \\
\mathscr{D S}-\underline{\underline{\underline{Z}}\left\{S^{2}\left[\mathscr{D}_{z}-\frac{3}{2}(Q / S)_{2}^{2}\right]\right\}_{z}=}=-S P_{z}-\underline{\frac{1}{8} C\left[S^{2}(Q / S)_{z z}\right]_{z z}}+\underline{3 C\left[S(Q / S)_{z}\right]_{z}}
\end{gathered}
$$

$$
\begin{aligned}
& \mathscr{D}=\left[Q_{t}+\left(Q^{2} / S\right)_{z}\right] / S, \\
& P=4\left(2 S+S_{z}^{2}-S S_{z z}\right)\left(4 S+S_{z}^{2}\right)^{-3}+B o z .
\end{aligned}
$$

$B o=\rho g R^{2} / \sigma$ being the static Bond number, where $g$ is the acceleration due to the axial microgravity. If the underlined terms in (2.7) are neglected. the momentum equation reduces to $\mathscr{D}=-P_{z}$, which is the same as that obtained from the aforementioned slice model.

Boundary conditions are

$$
Q( \pm \Lambda . \iota)=0 . \quad S( \pm \Lambda, t)=1
$$

and initial conditions are

$$
Q(z, 0)=Q_{0}(z) . \quad S(z, 0)=S_{0}(z) .
$$

In addition. one more condition could be introduced. stating the overall mass conservation during the evolution. that is

$$
\int_{-A}^{+1} S \mathrm{~d} z=\int_{-1}^{+1} S S_{0} \mathrm{~d} z
$$

\section{Asymptotic expansions and self-similar solution}

The nature of the bifurcation appearing in cylindrical liquid bridges when $A=\pi$ indicates that if the slenderness of the liquid bridge differs in a small quantity $\epsilon \lambda$ from the critical ralue. $t$ the shape deformations to be expected are of order $\epsilon$ illartinez 1983). Thence. assuming that $Q=o\left(\epsilon^{n_{1}}\right)$ and introducing a new time $\tau=\epsilon^{n_{2}} t$ and a new viscosity parameter $c=\epsilon^{n}, r$. the following relations are obtained. From the continuity equation (?.6.6)

$$
\epsilon \epsilon^{n_{1}}: \sim \epsilon^{n_{1}} \text {. }
$$

+ The slenderness of the liquid loridge an be expressed as $.1=\pi(1+\epsilon \lambda)$. where 6 stands for the order of magnitude $(e \ll 1)$ and $d$ is a parameter of order unity giving the difference between the senderness of the bridge and the static stability limit. 
On the other hand. if the interface shape is not an equilibrium one. an order- $e^{3}$ capillary gradient arises. tending to start the movement, and then the momentum equation gives

$$
\epsilon^{n_{1}} \epsilon^{n_{2}} \sim \epsilon^{-n_{3}} \epsilon^{n_{1}} \sim \epsilon^{3}:
$$

therefore the following results are obtained: $n_{1}=1, n_{2}=\frac{1}{2} \cdot n_{3}=-\frac{1}{2}$.

In consequence the rariables $S$ and $Q$ can be expanded in the form

$$
\begin{aligned}
& S=1+\epsilon^{\frac{1}{2}} s_{1}+\epsilon s_{2}+\epsilon^{\frac{3}{2}} s_{3}+o\left(\epsilon^{2}\right), \\
& Q=\epsilon q_{2}+\epsilon^{3} q_{3}+o\left(\epsilon^{2}\right),
\end{aligned}
$$

whereas the new time and the new viscosity parameter are defined as

$$
\tau=\epsilon^{\frac{1}{2} t} . \quad c=\epsilon^{-\frac{1}{2}} C^{\prime}:
$$

furthermore. a new spatial coordinate is introduced to normalize boundary conditions:

$$
x=\frac{z}{1+\epsilon \lambda} \text {. }
$$

If the liquid bridge has a non-cylindrical volume the undisturbed interface shape will be $S=1+\epsilon s_{0}$ instead of $S=1$, and (3.1) should be replaced by

where

$$
S=1+\epsilon^{\frac{1}{2}} s_{1}+\epsilon\left(s_{0}+s_{2}\right)+\epsilon^{3} s_{3}+o\left(\epsilon^{2}\right),
$$

$$
s_{0}=v(1+\cos x) \text {, }
$$

as can be easily shown through an hydrostatic analysis; $v$ is related to the volume of the liquid bridge I" through

$$
r^{\circ}=2 \pi \Lambda(1+\epsilon v)=2 \pi^{2}[1+\epsilon(\lambda+v)]+o\left(\epsilon^{2}\right) .
$$

On the other hand. in the case of a small gravity field acting parallel to the axis of the liquid bridge, Vega \& Perales (1983) calculated that the static stability limit depends on the Bond number as $B o^{3}$, and thence the following expansion can be used:

$$
B o=\epsilon^{\frac{3}{2}} b \text {. }
$$

Introducing (3.2)-(3.5) and (3.8) in (2.6)-(2.9) and conditions (2.10)-(2.12) and equating coefficients of equal power in $\epsilon$ yields the following set of problems: $\dagger$ order $\epsilon^{\frac{1}{2}}$

$$
\begin{gathered}
s_{1 x}+s_{1 x x x}=0, \\
s_{1}( \pm \pi, \tau)=0, \quad \int_{-\pi}^{\pi} s_{1}(x, \tau) \mathrm{d} x=0, \\
s_{1}(x, 0)=A_{0} \sin x,
\end{gathered}
$$

order $\epsilon$

$$
\begin{gathered}
s_{1 \tau}+q_{2 x}=0, \\
\left(s_{2}+s_{0}\right)_{x}+\left(s_{2}+s_{0}\right)_{x x x}-s_{1 x}\left(s_{1}+s_{1 x x}\right)=0, \\
s_{2}( \pm \pi, \tau)=0, \quad \int_{-\pi}^{\pi} s_{2}(x, \tau) \mathrm{d} x=0, \quad q_{2}( \pm \pi, \tau)=0, \\
s_{2}(x, 0)=0, \quad q_{2}(x, 0)=0,
\end{gathered}
$$

$\dagger$ To write down the different problems the initial conditions were $Q(\tilde{z}, 0)=0$ and $S(z, 0)=1+\epsilon^{\frac{1}{2}} A_{0} \sin x+\epsilon v(1+\cos x)$, which are similar to those used in numerical studies. Note that the interface perturbation $\epsilon^{\frac{1}{t}} A_{0} \sin x$ does not change the initial volume of the liquid bridge. 
order $\epsilon^{\frac{3}{2}}$

$$
\begin{aligned}
q_{2 \tau}-\frac{1}{8} q_{3 \tau x x}= & \frac{1}{2}\left(s_{3 x}+s_{3 x x x}\right)-\frac{1}{2}\left[s_{1 x}\left(s_{2}+s_{0}\right)_{x}\right]_{x} \\
& -\frac{1}{2}\left[s_{1}\left(s_{2}+s_{0}\right)\right]_{x}+\frac{1}{2} s_{1} s_{1 x}\left(s_{1}+s_{1 x x}\right) \\
& +s_{1 x}\left[\lambda+\frac{3}{8}\left(s_{1 x}^{2}-s_{1 x x}^{2}\right)\right]+c\left(3 q_{2 x x}-\frac{1}{8} q_{2 x x x x}\right)-b, \\
& s_{3}( \pm \pi . \tau)=0, \quad \int_{-\pi}^{\pi} s_{3}(x, \tau) \mathrm{d} x=0, \\
& s_{3}(x, 0)=0,
\end{aligned}
$$

where $s_{0}$ is given by expression (3.6).

The solution of (3.9) with conditions (3.10) and (3.11) is

$$
s_{1}=A_{1}(\tau) \sin x
$$

with $A_{1}(0)=A_{0}$, and the solution of the order- $\epsilon$ problem after replacing $s_{0}$ and $s_{1}$ by their expressions from (3.6) and (3.19) is

with $A_{1 \tau}(0)=0, A_{2}(0)=0$.

$$
\begin{gathered}
q_{2}=A_{1 r}(1+\cos x), \\
s_{2}=A_{2}(\tau) \sin x,
\end{gathered}
$$

The same procedure must be applied to the order $-\epsilon^{\frac{3}{2}}$ problem. The integration of (3.16) leads to

$$
\begin{aligned}
s_{3}=-\left[\frac{9}{8} A_{1 \tau \tau}+\frac{25}{8} c A_{1 \tau}-\left(\lambda-\frac{1}{2} v\right) A_{1}-\frac{3}{16} A_{1}^{3}\right] x \cos x \\
+\frac{1}{84}-A_{1}^{3} \sin 3 x+2\left(-A_{1 \tau \tau}+b\right) x+A_{3}(\tau) \sin x+A_{4}(\tau) \cos x ;
\end{aligned}
$$

the fulfilment of the conditions of anchorage to the disk edges yields $A_{4}=0$ and

$$
\frac{35}{8} \cdot A_{1 \pi}+\frac{75}{3} c A_{17}-\left(\lambda-\frac{1}{2} v\right) A_{1}-\frac{3}{16} \cdot A_{1}^{3}+2 b=0 \text {. }
$$

This equation determines the evolution of the amplitude of the interface deformation. together with initial conditions $A_{1}(0)=A_{0}$ and $A_{17}(0)=0$. already stated.

In the steady case this equation recovers the main features of the static analysis. For example. if the Bond number is zero the bifurcation $\frac{1}{2} x-\lambda=\frac{3}{16}+A_{1}^{2}$ is obtained. or. using non-expanded variables.

$$
\Lambda=\pi\left[1-\frac{3}{16}\left(1-S_{\mathrm{m}}\right)^{2}\right]+\frac{1}{2} \pi\left(\frac{V}{2 \pi \Lambda}-1\right) .
$$

$s_{m}$ being the minimum section (or the maximum. within this approximation) of the liquid bridge. As (3.2t) shows. when the liquid bridge has evlindrical rolume a bifurcation appears at $A=\pi$. If the colume enclosed in the liquid bridge is greater than the crlindrical one this phenomenon occurs at slendernesses greater than $\pi$. and. in contrast. if the volume is less than that of the cylinder the bifurcation appears at $\Lambda<\pi$.

If the Bond number is not equal to zero the bifureation points appear when $\left(\frac{26}{3} b\right)^{2}=\left[\frac{16}{9}\left(\frac{1}{2} r-\lambda\right)\right]^{3}$. as is well known from the cubic-equation solution. and the following stability limit results:

$$
A=\pi\left[1-\frac{3}{2}\left(\frac{3}{2} B o^{2}\right)^{\frac{1}{3}}\right]+\frac{1}{2} \pi\left(\frac{r^{*}}{2 \pi A}-1\right) .
$$

If $I^{\prime}=2 \pi .1$ the stability limit calculated by Vega \& Perales (198:3) is obtained. The volume plays the same role as in the preceding case.

Let us introduce $m \delta=\lambda-\frac{1}{2}$. Where $\delta$ is a positive parameter and $m$ takes aceount 
of the sign ( $m= \pm 1)$. Since a self-similar solution exists the parameter $\delta$ may be eliminated from (3.23). Redefining the remaining parameters and variables as

$$
A_{1}=4 x\left(\frac{1}{3} \delta\right)^{\frac{1}{2}}, \quad \tau=\frac{5}{2} \theta(2 \delta)^{-\frac{1}{2} .} \quad c=\frac{2}{5} \gamma(2 \delta)^{\frac{1}{2}} . \quad b=2 \delta \beta\left(\frac{1}{3} \delta\right)^{\frac{1}{2}} .
$$

(3.23) beromes similar to the I)uffing equation (Stoker 1966)

$$
x_{t \theta}+\gamma x_{\theta}-m x-x^{3}+\beta=0 \text {. }
$$

which. if a dot is used to indicate time derivative $(\dot{x}=\mathrm{d} \alpha / \mathrm{d} \theta)$. can be rewritten as

$$
\frac{\mathrm{d} \dot{x}}{\mathrm{~d} x}=\frac{m \alpha+\alpha^{3}-\gamma \dot{x}-\beta}{\dot{x}} .
$$

\section{Phase-plane analysis}

We can obtain an easy understanding of the particularities of the liquid-bridge problem through a phase-plane analysis. It is convenient to do this separately for the cases $\beta=0$ and $\beta>0$. In the first case (3.28) reduces to

$$
\frac{\mathrm{d} \dot{x}}{\mathrm{~d} x}=\frac{m \alpha+x^{3}-\gamma \dot{\alpha}}{\dot{x}} \text {. }
$$

To simplify the explanation let us denote the parameters involved in the problem with labels indienting their main physical meaning. so that in the following we refer to $x . \theta, \gamma$ and $\beta$ as deformation of the interface, time, viscosity and gravity respectively. The singular points of (4.1) always occur at $\alpha=0$. the values of $\alpha$ given hy the roots of $\alpha^{3}+m x=0$. If $m=1$ there is only one real root, $x=0$, which corresponds to a saddle point: the configuration is unstable.

If $m=-1$ threc singular points exist at $\alpha=0$ and $\alpha= \pm 1$. The singular points $\alpha= \pm 1$ are always saddle points (see figure 2 ), but the character of the singularity at the origin depends on the value of the viscosity of the liquid, although the behaviour is stable at $\alpha=0$ no matter the value of $\gamma$. If $\gamma \geqslant 2$ the origin is a stable node: the liquid bridge, when slightly disturbed, returns to the equilibrium configuration without any oscillation (this result has already been obtained by Meseguer (1983) through a linear analysis of the Cosserat model). When $\gamma<2$ the origin becomes a stable focus: if the initial perturbation is small enough the liquid bridge oscillates around the equilibrium shape with damping amplitude until the equilibrium is reached. The last case corresponds to an inviscid liquid bridge, $\gamma=0$, for which the origin is a centre, and pure oscillating evolutions around the equilibrium shape are obtained.

If an axial microgravity is considered $(\beta>0)$ the singular points are also placed at $\dot{\alpha}=0$. and are given by the roots of $\alpha^{3}+m \alpha-\beta=0$. The number of real roots of this equation depends on the sign of $D=\left(\frac{1}{3} m\right)^{3}+\left(\frac{1}{2} \beta\right)^{2} ; m=1$ implies $D>0$ and only one real root $\alpha_{1}<0$, which corresponds to a saddle point (the configuration is unstable). When $m=-1$ the sign of $D$ is determined by the value of microgravity. If $\beta>2 / \sqrt{ } 27$ the discriminant $D$ is positive and the same thing happens as in the preceding case. When $\beta=2 / \sqrt{ } 27$ the limit of stability is reached; there are two rea] roots $\alpha_{1}<0 . \alpha_{2}>0$ ( $\alpha_{2}$ being a double root), but the behaviour remains unaltered: the liquid column is unstable. Finally, if $\beta<2 / \sqrt{ } 27$ there are three real roots $\alpha_{1}<0$. $\alpha_{3}>\alpha_{2}>0$; from these the extreme singular points are saddle points, whereas the middle one behaves as in the $\beta=0$ case, and a node, a focus or a centre is obtained depending on the value of viscosity $\gamma$. 

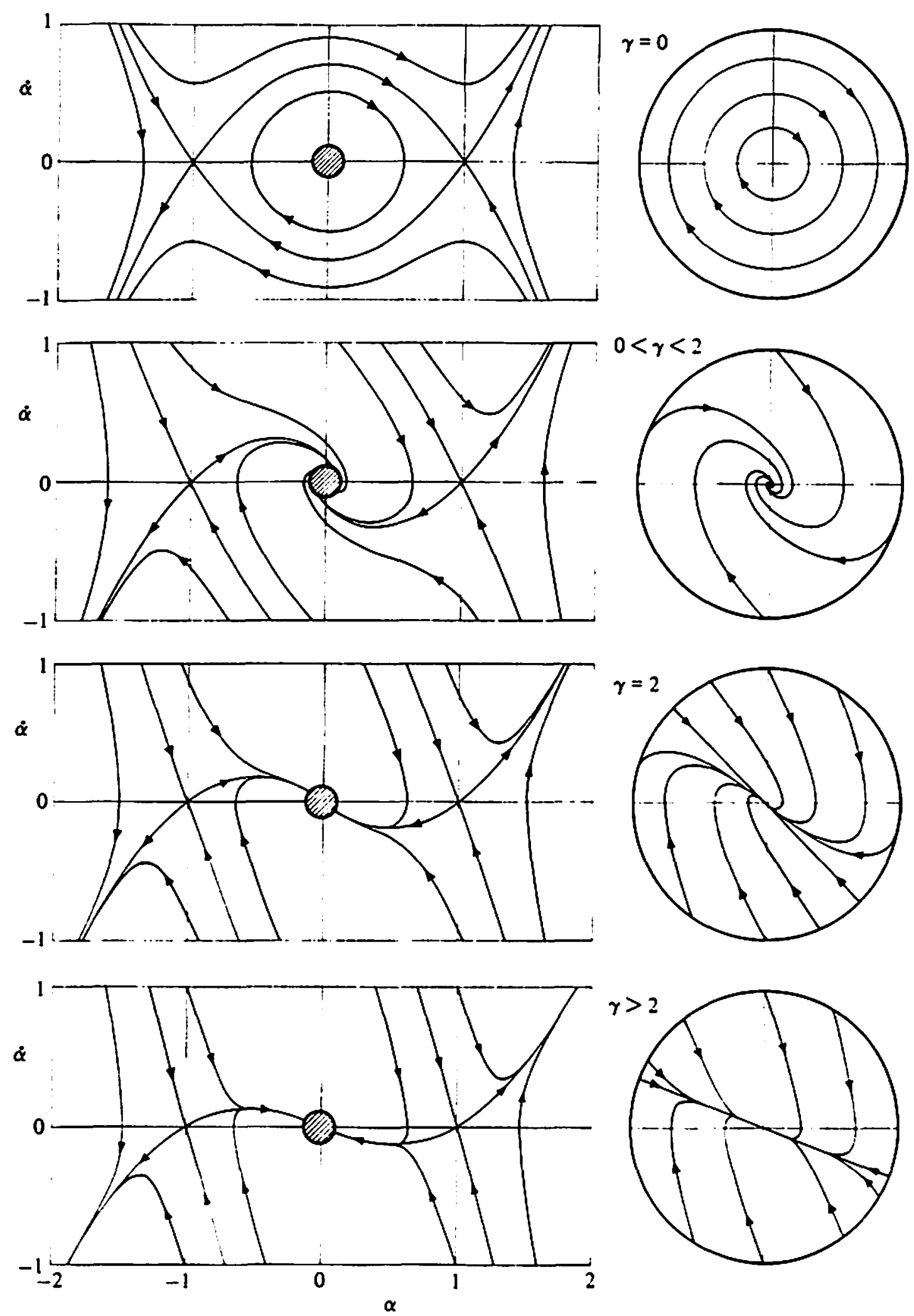

Fiavre 2. Phase plane of (3.28) in the case $\beta=0, m=-1$. The different sket ches show the influence of the self-similar parameter of viscosity $\gamma$ on the behaviour of the liquid bridge. Additional details of the phase plane in the vicinity of the origin are given in the inserts placed at the right-hand side.

Additional insight on the liquid-bridge behaviour can be obtained by plotting the variation with the interface deformation of the energy of the liquid bridge in the steady case. This energy will be the potential energy

$$
E_{\mathrm{p}}=\pi B o \int_{-A}^{A}(z+A) F^{2} \mathrm{~d} z=\pi b \epsilon^{\mathrm{z}}(1+\epsilon \lambda)^{2} \int_{-\pi}^{\pi}(x+\pi) S \mathrm{~d} x
$$



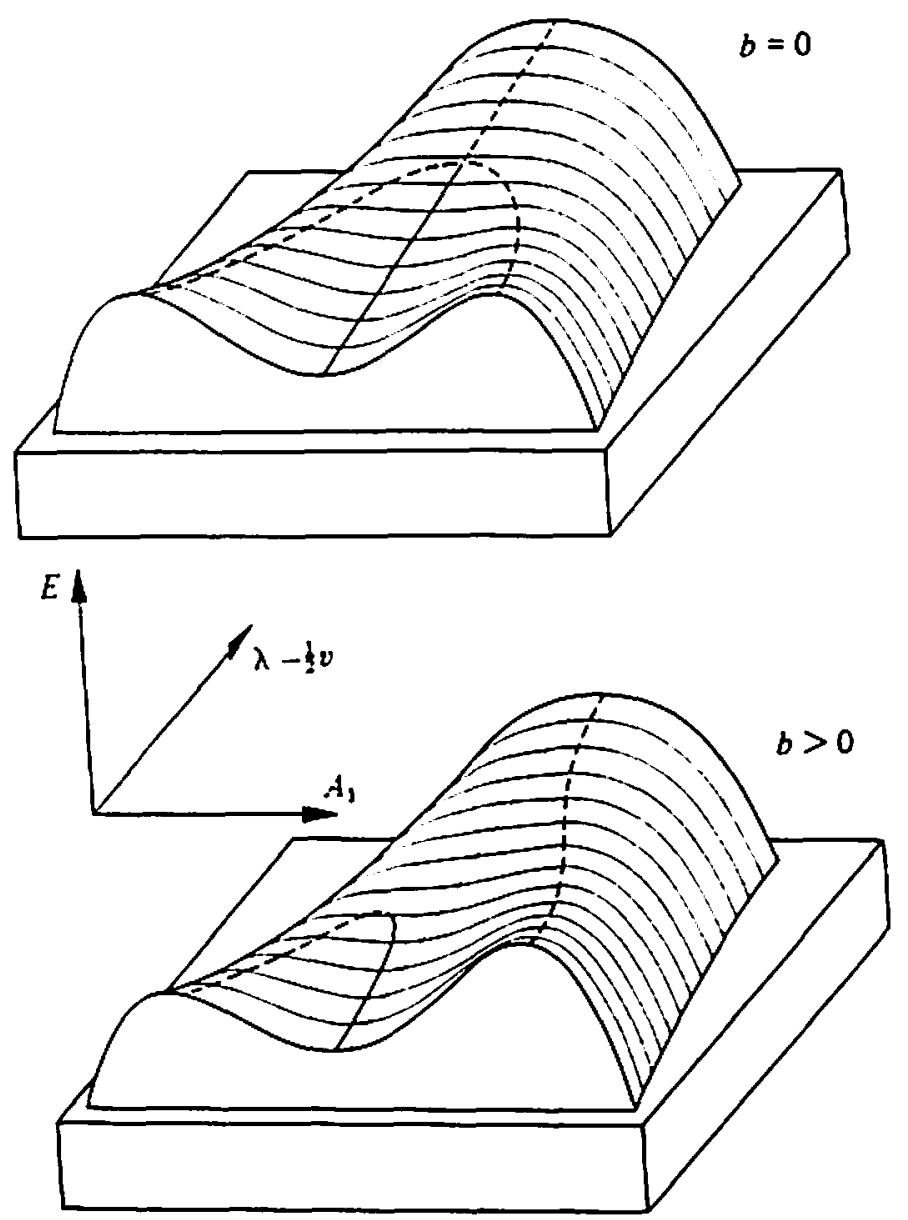

Fu:l'k: 3. Variation of the liçuid-bridge energy $E$ with expanded interface perturhation $A$, and reduced slenderness $\lambda-\frac{1}{2} r$. Thick solid lines indicate the stable equilibrium configurations, whereas dashed lines indicate the unstable ones. The thin solid line shown in the $b>0$ sketch corresponds to cylindrical configurations.

plus the energy associated with the free surface

$$
E_{\mathrm{s}}=2 \pi \int_{-\Lambda}^{\Lambda} F\left(1+F_{z}^{2}\right)^{\frac{1}{2}} \mathrm{~d} z=2 \pi(1+\epsilon \lambda) \int_{-\pi}^{\pi}\left[S+\frac{1}{4}(1+\epsilon \lambda)^{-2} S_{x}^{2}\right]^{\frac{1}{2}} \mathrm{~d} x
$$

Integration of (4.2) and (4.3), retaining terms up to order $\epsilon^{2}$. vields

$$
\begin{aligned}
E= & E_{\mathrm{p}}+E_{\mathrm{s}} \\
= & 4 \pi^{2}\left\{1+\epsilon\left(\lambda+\frac{1}{2} v^{v}\right)+\frac{1}{2} \epsilon^{3} \pi b\right. \\
& \left.-\frac{1}{8} \epsilon^{2}\left[r^{2}-4 \lambda v+\left(\lambda-\frac{1}{2} v\right) A_{1}^{2}+\frac{3}{32} A_{1}^{4}-4 b A_{1}\right]\right\}+o\left(\epsilon^{3}\right) .
\end{aligned}
$$

Equilibrium shapes are reached when $\partial E / \partial A_{1}=0$, that is, when

$$
\left(\lambda-\frac{1}{2} v\right) A_{1}+\frac{3}{16} A_{1}^{3}-2 b=0 .
$$

Obviously. this expression coincides with that obtained from (3.23) in the steady case. In figure 3 the variation with $A_{1}$ and $\lambda-\frac{1}{2} v$ of the liquid-bridge energy in the region close to the static stability limit is plotted in both cases $b=0$ and $b>0$. After these plots. liquid-bridge behaviour becomes similar to that of a marble which is constricted to move on the energy surface. If both the slenderness and the volume of the liquid bridge are kept constant the marble will be restricted to move along the lines $\lambda-\frac{1}{2} v=$ constant, the viscosity playing the role of friction. 


\begin{tabular}{|c|c|c|c|c|}
\hline$m$ & $\alpha_{0}$ & $\theta$ & $\psi_{i}, \phi_{i}$ & Comments \\
\hline+1 & & $\theta=\left(\alpha_{0}^{2}+1\right)^{-1} F\left(\psi_{1}, \phi_{1}\right)$ & $\begin{array}{l}\psi_{1}=\sin ^{-1}\left[\left(\alpha_{0}^{2}+2\right) /\left.\left(2 \alpha_{0}^{2}+2\right)\right|^{1}\right. \\
\phi_{1}=\cos ^{-1}\left(\alpha_{0} / x\right)\end{array}$ & $\begin{array}{c}\text { Breaking } \\
\alpha^{2}>\alpha^{2}\end{array}$ \\
\hline-1 & $\begin{array}{l}\alpha_{0}^{2}>2 \\
\alpha_{0}^{2}=2\end{array}$ & $\begin{array}{l}\theta=\left(\alpha_{0}^{2}-1\right)^{-\frac{1}{2}} F\left(\psi_{2} \cdot \phi_{1}\right) \\
\theta=\frac{1}{2} \sqrt{ } 2 \cos ^{-1}(2 / \alpha)\end{array}$ & $\psi_{2}=\sin ^{-1}\left[\left(\alpha_{0}^{2}-2\right) /\left(2 \alpha_{0}^{2}-2\right)\right]^{\frac{1}{2}}$ & \\
\hline & $1<x_{0}^{2}<2$ & $\theta=\frac{\sqrt{ } \mathbf{2}}{\alpha_{0}}\left[F\left(\psi_{3}, \frac{1}{2} \pi\right)\right.$ & $\psi_{3}^{\prime}=\sin ^{-1}\left[\left(2-x_{0}^{\hat{2}}\right) / \alpha_{0}^{2}\right)^{\frac{1}{2}}$ & \\
\hline & $0<\alpha_{0}^{2}<1$ & $\begin{aligned} \theta= & \left.-F\left(\psi_{3}, \phi_{3}\right)\right] \\
& \left.-F\left(\psi_{4}, \phi_{4}\right)\right]\end{aligned}$ & $\begin{array}{l}\phi_{3}=\sin ^{-1}\left(\alpha_{0} / x\right) \\
\psi_{1}=\left.\sin ^{-1}\left[\alpha_{0}^{2} /(2)-\alpha_{0}^{2}\right)\right|^{\frac{1}{2}} \\
\phi_{4}=\sin ^{-1}\left(\alpha / \alpha_{0}\right)\end{array}$ & $\begin{array}{c}\text { Oscillation } \\
x^{2}<x_{0}^{2}\end{array}$ \\
\hline \multicolumn{5}{|c|}{$F$ stands for the elliptic integrals of the first kind (see (5.2)) } \\
\hline \multicolumn{5}{|c|}{ TABLE 1} \\
\hline
\end{tabular}

\section{Self-similar results}

Since there are two parameters involved in (3.27). viscosity $\gamma$ and axial microgravity $\beta$, we will analyse separately the effects of each of them. Integration of (3.27) allows us to calculate the evolution time once the initial conditions are specified (for instance $\alpha(0)=x_{0}, x_{\theta}(0)=0$, as stated above).

In the case of an inviscid liquid bridge $(\gamma=0)$ a first integration of (3.28) leads to

$$
\theta=\int_{x_{0}}^{\alpha} \frac{\mathrm{d} \alpha}{\left(\left(\alpha^{2}-\alpha_{i 1}^{2}\right)\left(m+\frac{1}{2}\left(\alpha^{2}+x_{0}^{2}\right)\right)-\left.2 \beta\left(\alpha-x_{0}\right)\right|^{\frac{1}{2}}\right.} .
$$

which in turn may be expressed in terms of elliptic integrals of the first kind

$$
F(\psi, \phi)=\int_{0}^{\phi} \frac{d \phi}{\left(1-\sin ^{2} \dot{\psi} \sin ^{2} \phi\right)^{\frac{1}{2}}} .
$$

Breaking time will be reached when the amplitude of the deformation of the interface becomes infinite. or. in other words. when the perturbation increases, ceases to be of order $\epsilon^{\frac{1}{t}}$ and becoming of order unity. On the other hand. sscillation periods are four times the time spent by the liquid bridge to reduce the amplitude of the interface deformation from its initial value $\alpha=x_{0}$ to $\alpha=0$.

When no microgravity is considered $(\beta=0) \psi$ and $\phi$ can be easily related to $\alpha$ and $x_{0}$. In table 1 the different expressions to be used in this case depending on the ralues of both $m$ and $x_{0}$ are shown. In figure $t$ the self-similar variables, breaking time $\theta_{b}$ and oseiliation period $\theta_{\mathrm{T}}$. versus the initial deformation of the liquid bridge $\alpha_{0}$ are plotted. The evolution time increases as the initial perturbation approaches the unstable equilibrium shapes $\left(\alpha_{0}= \pm 1\right.$ if $m=-1$ and $\alpha_{0}=0$ when $m=1$ ) where the evolution time becomes infinite.

When microgravity is not zero the relationships between is. $\phi$ and $x . x_{0}$ are not as simple as indicated in table 1 . and it is preferable to integrate (3.27) directly by using some numerical method. In our aase this integration has been performed by. means of a fourth-order variable-step Runge-hutta method. The results obtained are plotted in figure 5 . which shows the influence on the breaking time of an axial microgravity. Breaking time decreases as $\beta$ increases. the intluence of $\beta$ being greater as the initial deformation is smaller. For a griven value of $\alpha_{0}$ the slope of the corresponding curve decrease's with $\beta$ : this phenomenon cen be explained as owing to inertia effects. which become more and more important as the erolution time decreases. 

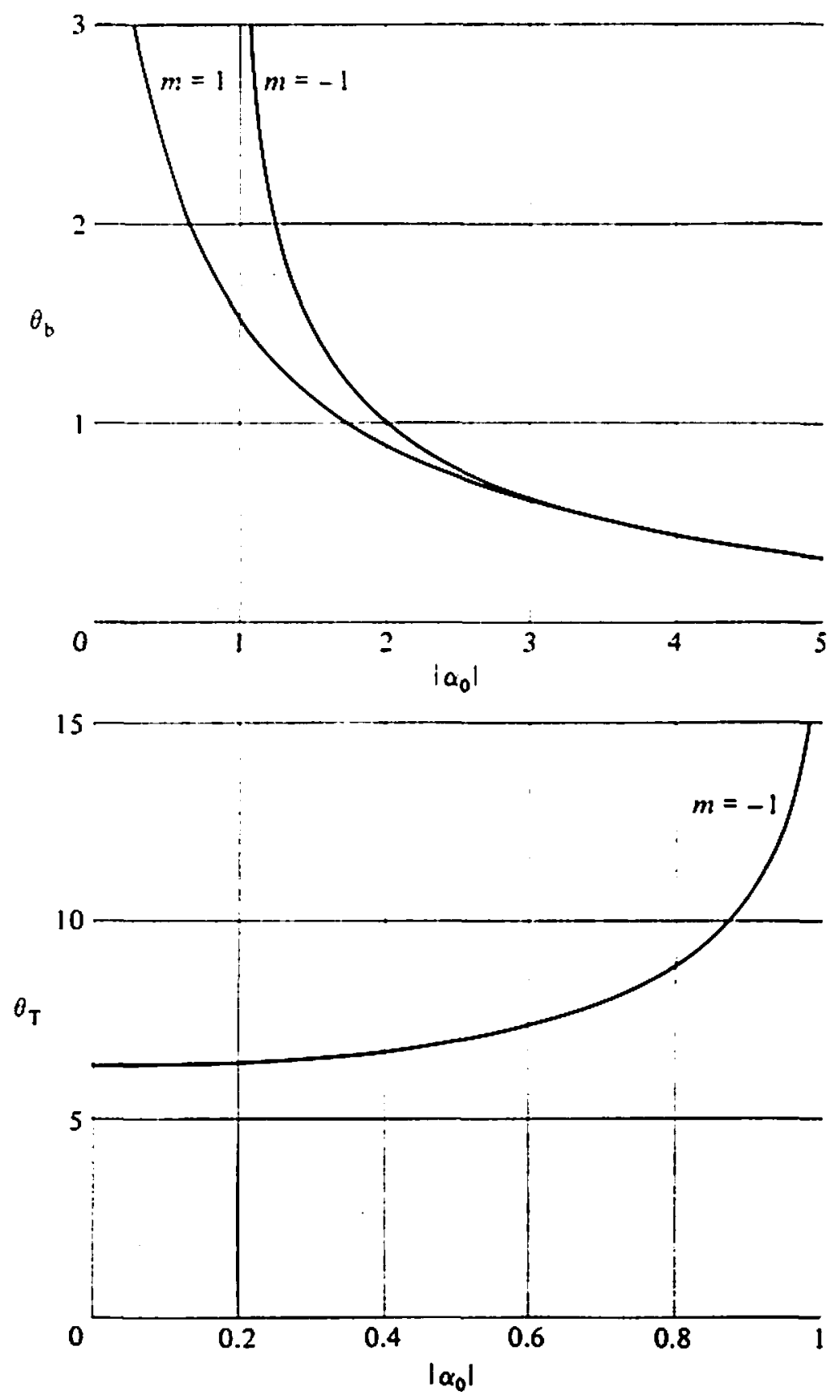

Figtre 4. Erolution of the liquid bridge in self-similar rariables. Breaking time $\theta_{\mathrm{b}}$ and oscillation period $\theta_{\mathrm{T}}$ versus initial deformation $\alpha_{0}$ in the case of an inviscid liquid bridge under gravitationless conditions $(\gamma=0, \beta=0)$.

Concerning viscosity effects, the results obtained by numerical integration of (3.27) in the case $\beta=0, \gamma>0$ are plotted in figure 6 . Also in this case the results are in complete agreement with those one could expect from experimental evidence: the viscosity increases the breaking time, the rate of increase being greater as $\alpha_{0}$ is smaller.

\section{The one-dimensional slice model}

To conclude this analytical study of the dynamics of liquid bridges it seems convenient to compare results obtained through this perturbation analysis with those obtained by numerical integration of the complete equations of the nonlinear one-dimensional slice model. As stated in §2, the slice model is generated by deleting 


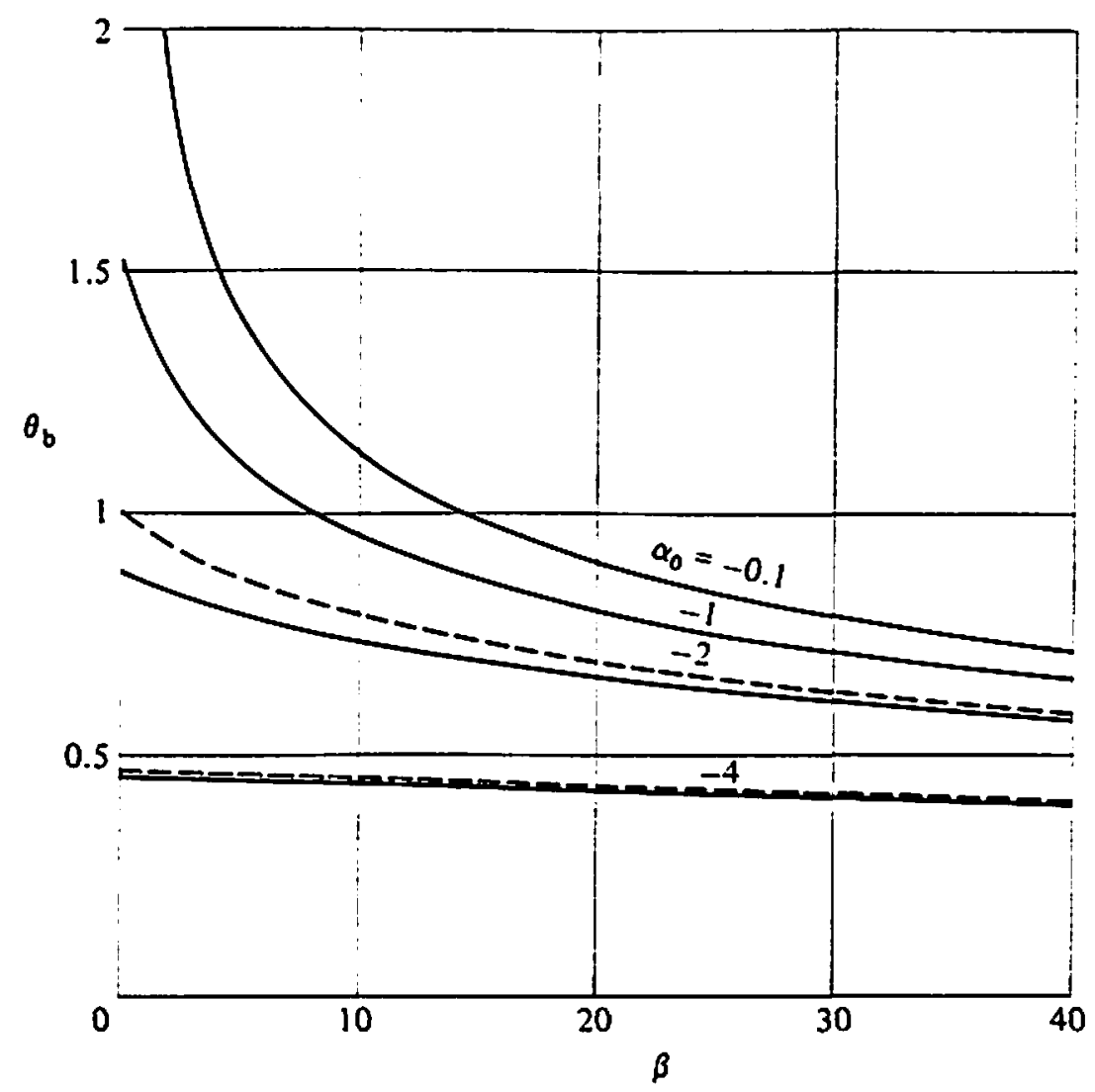

Frgure 5. Evolution of the liquid bridge in self-similar variables. Breaking time $\theta_{0}$ versus microgravity $\beta$ in the case of inviscid liquid bridges $(\gamma=0)$. Solid lines correspond to $m=1$ and dashed lines to $m=-1$. Numbers on the curves indicate the value of the initial deformation $\alpha_{0}$. In this plot only negative values of $\alpha_{0}$ are considered (see also tigure 3 ).

the underlined terms in (2.7). From there on. developments are similar to those explained in $\S 3$, yielding the following equation:

$$
3 A_{1 \pi}-\left(\lambda-\frac{1}{2} v\right) A_{1}-\frac{3}{16} \cdot A_{1}^{3}+2 b=0 .
$$

which replaces (3.23). Stability limits are the same in both the Cosserat and slice models. Introducing self-similar variables like (3.26) in (6.1) leads to

$$
\alpha_{\theta \theta}-m \alpha-\alpha^{3}+\beta=0 .
$$

which is the same as (3.27) in the case $\gamma=0$; the only difference arises in (3.26). in which $\tau=\theta(3 / \delta)^{\frac{1}{2}}$ would be used instead of $\tau=\sum_{2}^{5} \theta(2 \delta)^{-\frac{1}{2}}$. Thence. within the approximation of this perturbation analy'sis. the evolution time $\tau_{\mathrm{C}}$ obtained through the inviscid one-dimensional Cosserat model becomes $2 \%$ greater than that obtained through the one-dimensional slice model. $\tau_{\mathbf{S}}$ :

$$
\tau_{\mathrm{C}}=\frac{5}{2 \vee 6} \tau_{\mathrm{S}}
$$

Available numerical results refer to the evolution of eylindrical inviscid liquid bridges in gravitationless conditions $(v=0, \gamma=0, \beta=0)$ : in consequence. the analytical evolution time will be given by the expressions given in table 1.

The main discrepancy between the numerical and analytical results arises in the self-similarity. In effect. the numerical results do not reduce to one single curve when expressed in self-similar variables. To explain this disagreement we must take into account the fact that the ralidity of the analytical analysis is supported by the 


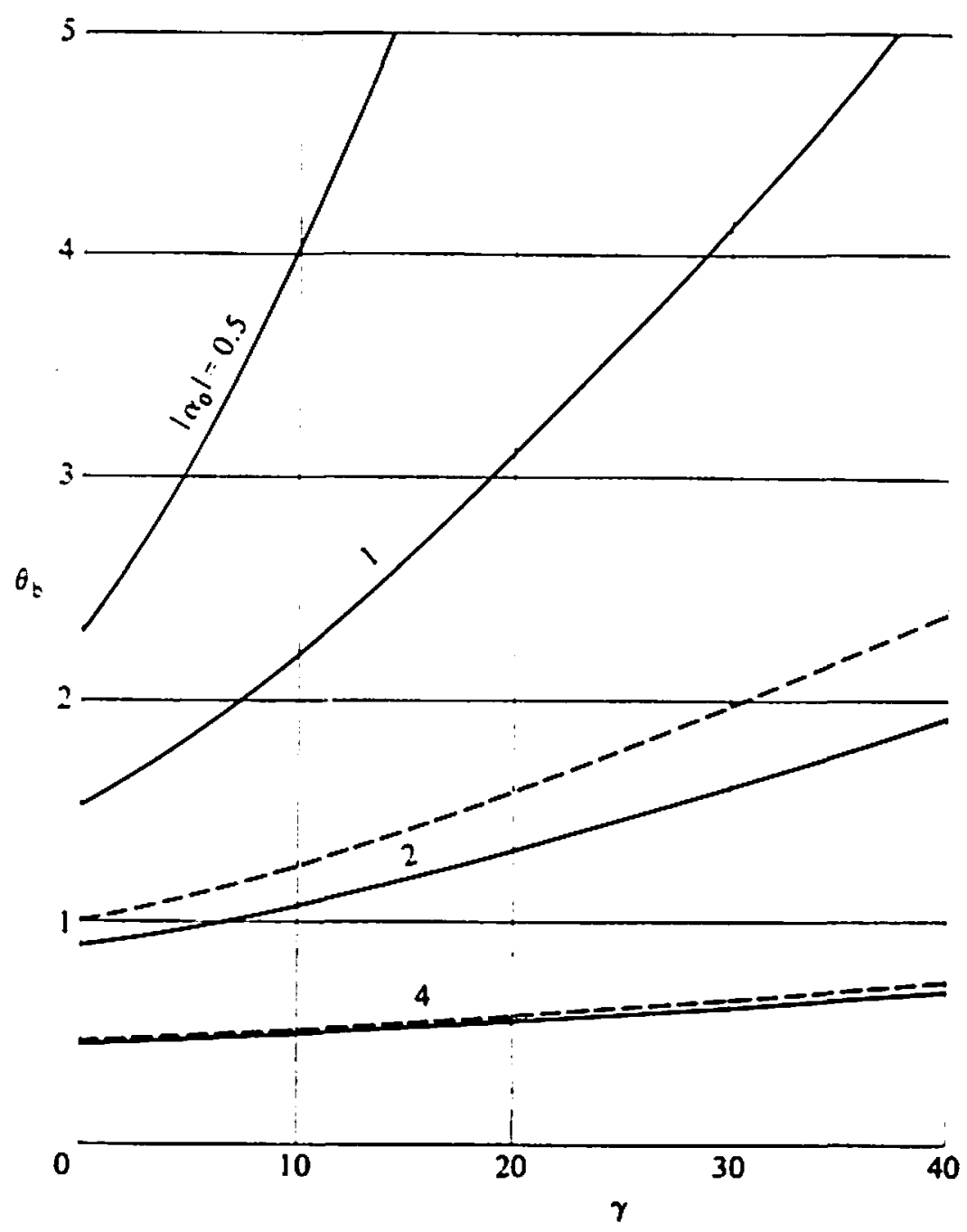

Figcre 6. Evolution of the liquid bridge in self-similar variables. Breaking time $\theta_{\mathrm{b}}$ versus viscosity $\gamma$ in the case of liquid bridges under gravitationless conditions $(\beta=0)$. Solid lines correspond to $m=1$ and dashed lines to $m=-1$. Numbers on the curves indicate the value of the initial deformation $\alpha_{0}$.

hypothesis that the perturbations are small enough, whereas numerical results have been obtained for values of the initial deformation of the interface $1-S_{m}$ ranging between 0.19 and 0.64 .

Using physical variables, the variation with slenderness of both breaking time and oscillation period of liquid bridges whose initial deformation is $1-S_{\mathrm{m}}=0.19$ are shown in figure $\overline{7}$. The relative difference observed between both analytical and numerical curves (greater in the case of breaking) suggests a revision of the definition of breaking time introduced in $\S 5$. Breaking time is reached when $\alpha$ becomes infinite; however, some care is needed when this process is considered, because, if the interface deformation ceases to be of order $\epsilon^{\frac{1}{2}}$ and becomes larger, higher-order terms in the asymptotic expansion would not be negligible, contributing to a fast variation of the interface deformation and reducing the evolution time.

If numerical results are extrapolated to lower values of $1-S_{m}$ then the difference between the analytical and numerical results decreases as one approaches the static stability limit; for instance, in the case of oscillation this difference may be neglected if the slenderness of the liquid bridge is greater than 3.1, and this value of the slenderness could be a lower bound of the region in the neighbourhood of the limit of stability for which analytical results are applicable.

In conclusion. this perturbation analysis seems to be a powerful tool in studying the behaviour of liquid-bridge configurations close to the stability limit, just in that 


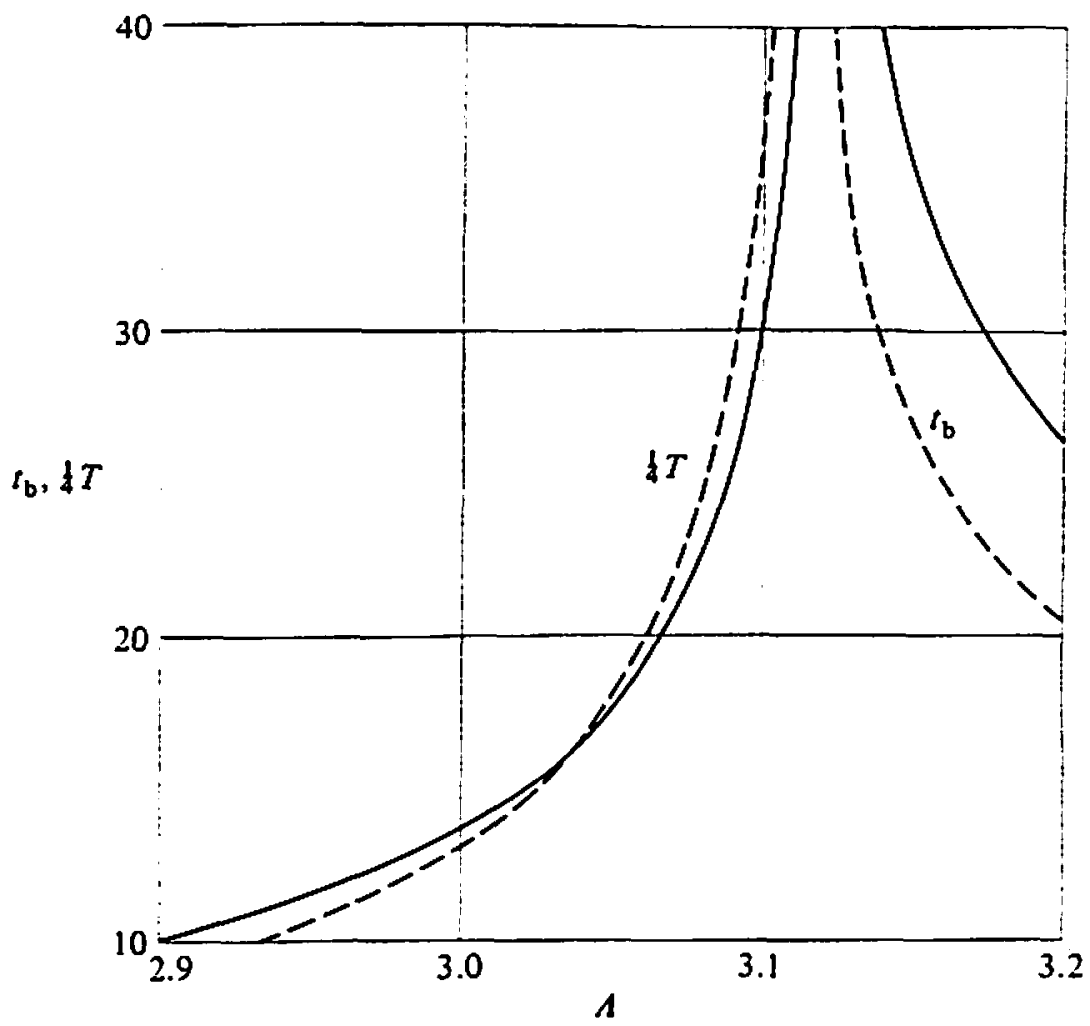

Figure 7 . Non-dimensional breaking time $t_{\mathrm{b}}$ and oscillation period $T$ versus slenderness $A$ in the case of inviscid liquid bridges under gravitationless conditions $(C=0 . B o=0)$ with an initial deformation $1-S_{\mathrm{m}}=0.19$. Solid lines correspond to results obtained from the perturbation analysis whereas dashed lines correspond to numerical results (Meseguer 1983). In this plot one-(quarter of the oscillation period (curves on the left-hand side) has been plotted instead of the whole period because of scale constraints.

region where computing methods become inappropriate. However. two main constraints must be pointed out: first. the difficulty in extending the perturbation analysis to liquid bridges having volume greatly different to that of the eylindrical one, owing to the complexity associated with the capillary-pressure term. and, secondly. that within the actual approximation the analytical method does not provide information on the volume of the two drops into which the liquid column splits once breaking takes place.

This work has been supported by the Spanish National Committee for Space Research (CONIE). The authors are indebted to Prof. A. Liñan for helpful disrussions and inspiration.

\section{REFEREXI:BS}

Bogy. D. B. 1979 Drop formation in a circular liquid jet. Ann. Rer. Fluid Mech. 11. 207-228. Gress. A. E. 1976 On the non-linear behariour of fluid jets. Indl J. Engng Sri. 14. t(t-633.

Lee. H. C. 1974 Drop formation in a liquid jet. IB.IJ J. Res. Der. 18. 364-369.

Martixez. I. 1983 stability of axisỵmetric liquid bridgess. In ES.A sipecial SP-191. pp. 2067-273. Paris: EsA.

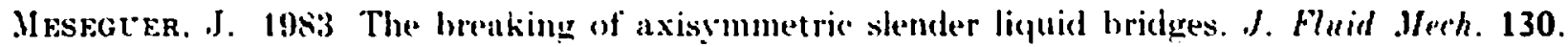
$12: 3-151$.

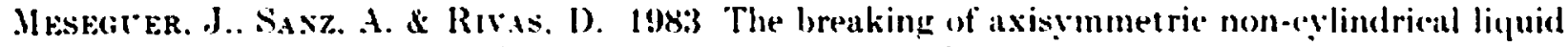
bridges. In ENA sperial SP.191. pp. $2(t)-263$. Paris: ESA.

Stokik. .J. J. 196ti Lomlinear librations. vol. II. Interscience.

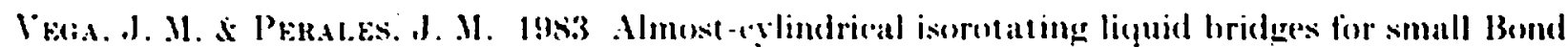

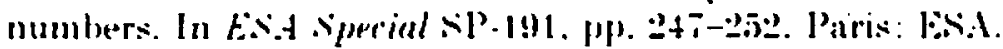

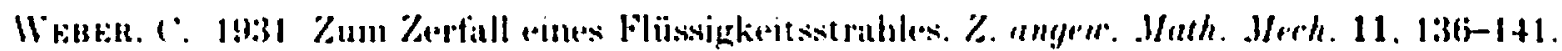

